\title{
ARRANJOS ESPACIAIS E AGRUPAMENTOS DE CRIANÇAS PEQUENAS EM CRECHES*
}

\section{SPATIAL ARRANGEMENTS AND GROUPS OF YOUNG CHILDREN IN DAY CARE CENTERS}

\author{
Renata Meneghini ${ }^{1}$ \\ Mara I. Campos de Carvalho ${ }^{2}$
}

MENEGHINI, R; CAMPOS-DE-CARVALI IO, M. I. Arranjos Espaciais e Agrupamemtos de Crianças Pequenas em Creches. Rev Bras. Cresc. Desenv. Hum., S. Paulo, 7 (1), 1997.

Resumo: Este trabalho verificou a formação de agrupamentos em duos, trios, quadras e com cinco ou mais elementos, utilizando a mesma coleta de dados do estudo anterior, realizado com crianças de 2-3 anos de duas creches da região de Ribeirão Preto (SP) que atendem famílias de baixa renda. Os dados foram obtidos por duas câmeras fotográficas com funcionamento automático e simultâneo a cada 30 segundos, em três fases: I - arranjo aberto: habitual (4 sessões): II - arranjo aberto: introdução de pequenas estantes de madeira nas laterais (6 sessões); III - arranjo semi-aberto: montagem de duas zonas circunscritas (6 sessões). Proximidade física (distância de até $1 \mathrm{~m}$ ) foi utilizada para registrar os agrupamentos, verificando-se: (1) maior estruturação espacial acarreta acréscimo na ocorrência de agrupamentos entre crianças e redução daqueles com o adulto; (2) maior freqûência de díades; (3) maior ocorrência de agrupamentos. especialmente com três ou mais crianças, em áreas mais estruturadas de cada fase. Salienta-se a importância do arranjo espacial na formação de agrupamentos infantis e no planejamento de ambientes infantis coletivos.

Palavras-chave: agrupamentos imfantis, arranjo espacial, creches, proximidade física.

\section{INTRODUÇÃO}

De acordo com nossa concepção, a creche - instituição que vem sendo cada vez mais utilizada em nosso meio - constitui um contexto de desenvolvimento de crianças pequenas, diverso tanto do lar como da escola. Neste contexto, um adulto cuida simultaneamente de várias crianças, onde os parceiros mais disponíveis para interação são outras crianças, geralmente coetâneos. Consideramos as interações entre crianças como tão importantes quanto as interações adulto-criança para o desenvolvimento infantil.

Nosso ponto de vista baseia-se em uma visão sistêmica de desenvolvimento. que enfatiza a relação bidirecional entre pessoa-ambiente (BRONFENBRENNER, 1977; BRONFENBRENNER \& CROUTER, 1983; CAMPOS-DE-

\footnotetext{
* Monografia defendida em maio/96 como conclusão do Programa de Bacharclado do Depto. de Psicologia e Educação da Faeuldade de Filosofia, Ciêneias e Letras de Ribeirão Preto - USP, apresentada pelo $1^{\circ}$ autor sob orientação do $2^{\circ}$. Trabalho apresentado na XIV ${ }^{\text {th }}$ Biennial Meethings of the International Society for the Study of Behavioural Dcvelopmcnt, agosto de 1996. Québec, Canadá.

1 Bolsista de iniciação Cientifica do CNl’q.

2 Profa. Dra. do Depto. de Psicologia e Educação da Faculdade de Filosofia, Ciências e Letras de Ribeirão Preto USP. Depto. de Psieologia e Edueação - Faeuldade de Filosofia, Ciências e Letras de Ribeirào Preto Universidadc dc São Paulo. End.: Av. dos Bandeirantes 3.900 - Ribeirão Preto - São Paulo - CEP 14040-901 - Tel. (016) 602-3661 - Fax (016) 602-3632 E-mail: miedear@usp.br
} 
CARVALHO, 1993; MOORE, 1987; STOKOLS, 1978; VALSINER, 1987; WOHLWILL, 1980; WOHLWILL \& HETT, 1987). Por um lado, a criança participa ativamente de seu desenvolvimento através de suas relações com o ambiente (especialmente pelas suas interações com adultos e demais crianças), inserido em um contexto sócio-histórico específico. A criança explora descobre e inicia ações em seu ambiente; seleciona parceiros, objetos, equipamentos e áreas para a realização de suas atividades, mudando o ambiente através de seus comportamentos. Por outro lado, os comportamentos infantis são influenciados pelo ambiente organizado pelos adultos, de acordo com seus objetivos pessoais construídos com base tanto em suas experiências anteriores, como nas concepções culturais sobre educação e desenvolvimento infantil.

A perspectiva de que as interações entre crianças são tão importantes quanto as interações adulto-criança para o desenvolvimento infantil, suscita questões sobre como os ambientes podem favorecer ou dificultar a ocorrência de interações, principalmente entre crianças pequenas em ambientes coletivos. tais como em creches.

Vários estudos têm demonstrado a importância de aspectos físicos do ambiente, tal como o papel dos objetos para interações de crianças (ECKERMAN \& STEIN. 1982; MUELLER \& LUCAS, 1975; STAMSAK \& VERBA, 1986). Porém, poucas pesquisas têm documentado a influência nos comportamentos infantis de espaços abertos e fechados, relativos à ausência ou presença de barreiras na área de atividades, particularmente em creches (LEGENDRE, 1983, 1987, 1989; LEGENDRE \& FONTAINE, 1991; MOORE, 1983, 1987).

O arranjo espacial é uma das variáveis do ambiente físico, que diz respeito à maneira como móveis e equipamentos existentes em um local posicionam-se entre si. LEGENDRE (1983, 1987, 1989; LEGENDRE \& FONTAINE, 1991) têm descrito as características de três tipos de arranjos espaciais e sua interdependência com as interações de crianças de 2-3 anos em creches francesas. O arranjo semi-aberto é caracterizado pela presença de zonas circunscritas, proporcionando à criança uma visão de todo o local. Zonas circunscritas são áreas delimitadas pelo menos em três lados por barreiras formadas por mobiliários, parede, desnível do solo, etc.; a caracteristica primordial destas zonas é a sua circunscrição ou fechamento, independentemente do tipo de material colocado para as crianças manipularem, o que, então, as diferenciam dos chamados cantos de atividades. Neste arranjo, as crianças ocupam preferencialmente as zonas circunscritas, nas quais ocorrem interações afiliativas freqüentes entre elas; suas aproximações do adulto, embora menos freqüentes, tendem a evocar mais respostas deste em comparação com outros arranjos. No arranjo aberto há ausência de zonas circunscritas, geralmente havendo um espaço central vazio. As interações entre crianças são raras, as quais tendem a permanecer em volta do adulto, porém ocorrendo pouca interação com o mesmo. Afora esta tendência, as crianças se espalham pela sala, com deslocamentos freqüentes. No arranjo fechado há a presença de barreiras físicas, por exemplo um móvel alto, dividindo o local em duas ou mais áreas, impedindo uma visão total do local. As crianças tendem a permanecer em volta do adulto, evitando áreas onde a visão do mesmo não é possível; há ocorrência de poucas interações entre crianças.

Faz-se necessário salientar que, mesmo quando as crianças se encontram dentro de uma zona circunscrita, elas devem poder visualizar facilmente o adulto. Este é um aspecto primordial para crianças de 2-3 anos devido aos comportamentos de apego, cujo padrão, descrito por vários autores em amostras de diferentes culturas, indica que crianças naquela faixa etária exibem fortes comportamentos de apego, não somente para a mãe, mas também para outras figuras, tal como a educadora da creche (ROSSETTl FERREIRA, 1984). Como a busca de proximidade física e/ou visual com a figura de apego é freqüente, conseqüentemente as crianças tendem a não permanecer em áreas fora do contato visual com o adulto. Geralinente isso é observado no arranjo fechado, onde as crianças evitam permanecer em áreas, inclusive em zonas circunscritas, onde não é possível ver as educadoras, como mostrado por LECENDRE (^1983, 1987, 1989; LEGENDRE \& FONTAINE, 1991). Desta maneira, é importante que os elementos usados nas montagens das zonas circunscritas em um arranjo semi-aberto sejam baixos o suficiente para pennitirem um fácil contato visual com o adulto.

Os resultados de Legendre são relevantes para o contexto pouco estruturado de nossas creches que atendem população de baixa renda - tal como o número grande de crianças pequenas (por exemplo, 10 a 35 crianças entre 18 a 36 meses) sob a supervisão de um só adulto; escassez de mobiliário, objetos e equipamentos; ausência de zonas circunscritas. Este tipo de ambiente não fàvoiece interações, seja entre criança-adulto e, especialmente, entre crianças menores de três anos, período no qual as habilidades verbais e sociais estão se desenvolvendo.

Nossos estudos direcionam-se para a contribuição do arranjo espacial para a oportunidade 
de interações de coetâneos tanto enfie si como com o adulto. Utilizamos a metodologia denominada por BRONFENBRENNER (1977) de experimento ecológico. Dentro de uma perspectiva ou abordagem ecológica (CAMPOS-DE-CARVALHO, 1993), este modelo propõe a realização de manipulações sistemáticas de uma única variável, a que está sob investigação, mantendo-se os demais componentes ambientais presentes, ou seja, aquelas manipulações que ocorrem no interior do sistema ecológico, preservando-se assim o sistema de interdependência entre os componentes ambientais.

Em estudo anterior (CAMPOS-DE-CARVALHO, 1989, CAMPOS-DE-CARVALHO \& ROSSETTI FERREIRO, 1993), evidenciamos uma relação entre aranjo espacial e ocupação da área de atividades livres por crianças entre 2-3 anos de duas creches da região de Ribeirão Preto (SP) que atendem famílias de baixa renda. A coleta de dados, utilizando duas câmeras fotográficas com funcionamento automático e conjunto a cada 30 segundos, foi realizada em três fases: Fase I - arranjo aberto: espaço usual, amplo e vazio; Fase II - arianjo aberto: introdução de pequenas estantes de madeira na periferia da área; Fase III - arranjo semi-aberto: montagem de duas zonas circunscritas. A análise da distribuição espacial das crianças mostrou: (I) reorganização da ocupação do espaço a cada fase; (2) ocupação preferencial de áreas mais estruturadas a cada fase; (3) maior concentraçao de crianças em volta do adulto em arranjos com menor estruturação espacial.

Estes resultados suscitaram inicialmente uma nova análise das fotos (CAMPOS-DE-CARVALHO e col. 1996), para analisar a ocorrência de pares infantis em cada tipo de arranjo espacial - foi verificado que, à medida que o espaço se tornou inais estruturado, houve aumento das parcerias formadas entre crianças e redução das formadas com o adulto. O presente trabalho foi proposto em decorrência deste estudo. pois pudemos observar a presença, não tão infreqüente. de agrupamentos com mais de duas crianças. As seguintes questões foram levantadas sobre a formação de agrupamentos infantis: (1) haveria diferença no total de agrupamentos formados entre crianças e entre criança-adulto na medida que o espaço se tornou mais estruturado, da Fase I para a III? (2) haveria diferença nos agrupainentos formados em áreas específicas em cada fase?

Utilizando a coleta de dados do estudo de Campos-de-Carvalho (CAMPOS-DE-CARVALHO, 1989; CAMPOS-DE-CARVALHO \& ROSSETTI FERREIRA, 1993), o objetivo do presente trabalho foi analisar os agrupamentos ocorridos em cada fase daquele estudo, visando caracterizá-los em termos de tamanho (duos, trios, quadras e agrupamentos com cinco ou mais elementos) e de rreqüência (e ocorrência, buscando verificar a existência de diferenças ou semelhanças entre os arranjos espaciais.

\section{MÉTODO}

Visto que foi utilizada a mesma coleta de dados do estudo de CAMPOS-DE-CARVALHO (1989; CAMPOS-DE-CARVALHO \& ROSSETTI FERREIRA, 1993), primeiramente descrevereinos esta metodologia e, a seguir, o procedimento utilizado na análise de dados do presente estudo.

\section{Sujeitos}

Foram observados dois grupos de crianças entre 2 e 3 anos e suas respectivas educadoras, uma por grupo, de duas creches da região de Ribeirão Preto (SP) que atendem população de baixa renda. Em cada grupo, as crianças estavam familiarizadas entre si, com a educadora e com a creche. Em ambas as creches as educadoras recebiam pouca orientação e treinamento para desenvolver a função, principalmente na Creche 1 .

A Creche 1, localizada na periferia de Ribeirão Preto, apresentava condições precárias nas instalações sanitárias e na conservação do prédio (adaptado para creche), escassez de mobiliários, decoração, equipamentos, materiais e brinquedos, os quais geralmente eram veIhos e quebrados. Atendia crianças de 2 meses a 10 anos, a maioria filhos de inães solteiras e empregadas domésticas.

Na Creche 2, localizada em uma cidade pequena da região de Ribeirão Preto, havia boas condições de instalações físicas e sanitárias sendo o prédio construído especialmente para o funcionamento da creche; embora houvess nesta creche maior quantidade e variedade de materiais, equipamentos, decoração e brinquedos novos, estes estavam pouco disponíveis às crianças, especialmente durante os períodos de atividades livres. Atendia crianças de 3 meses 4 anos, a maioria advindas de famílias de bóias frias e de funcionários da usina de cana-de-açúcar.

Outra diferença marcante entre as duas creches diz respeito ao fato de que as crianças da Creche 1 eram subdvididas por grupo de idade, sendo que cada grupo permanecia a maior parte do dia em uma sala específica enquanto que na Creche 2 as crianças também eram subdivididas por grupo de idade porém havia rodízio na utilização das salas. Ademais, a Creche I era administrada por uma assistente social sendo que as de- 
mais dez funcionárias, especialmente as educadc ras, participavam na programação geral d creche. $\mathrm{O}$ funcionamento e programação d Creche 2 eram decididos pela administrador ( $2^{\circ}$ grau) e por duas técnicas, psicóloga e fonoaudióloga, com pouca participação do demais funcionários, 12 mulheres e 2 homens.

O Grupo I, que freqüentava a Creche 1, era formado por 22 crianças na Fase I do estudo, 8 meninas e 14 meninos, com idade que variavam de 18 a 35 ineses (idade inédia 26 ineses). Nas fases subseqüentes, ocorridas seis meses após a primeira devido a problemas técnicos, a composição do grupo havia mudado, restando somente duas crianças que também participaram da fase inicial, sendo formado por 13 crianças, 8 meninas e meninos, de 20 a 35 meses de idade (idade média: 26 meses). O Grupo 2, observado na Creche 2, era formado por 11 crianças na primeira fase do estudo, 7 meninas e 4 meninos com idades que variavam de 26 a 31 meses (idade média: 28 meses); nas fases subseqüentes, ocorridas três meses após a inicial, o grupo era composto por 13 criancas (presença de duas crianças que não participaram da primeira fase), 8 meninas e 5 meninos, de 30 a 34 meses (idade média: 31 meses).

\section{Local e Situnção}

A coleta de dados foi realizada durante períodos de atividades livres regularmente programados pelas creches. Os materiais utilizados pelas crianças, geralmente sucatas e/ou brinqüedos velhos da própria creche, eram levados pelas educadoras e colocados no centro do local. Em ambas as creches o local caracterizava-se por um espaço amplo e vazio, sem zonas circunscritas, definindo um arranjo espacial aberto, o qual foi modificado nas duas outras fases do estudo.

Na Creche 1, a coleta de dados foi realizada em um galpão coberto de aproximadamente $126 \mathrm{~m}^{2}$ de superfície. Este galpão continha uma plataforma de cimento (5,56 × 2,68 x 0,45 m) com um degrau de acesso, pilastras de concreto nas bordas laterais e um bebedouro de cimento inacessível aos sujeitos devido sua altura. As crianças utilizavam diariamente este galpão para a ocorrência de atividades livres, sendo que em sua sala privativa ocorriam principalmente atividades estruturadas pela educadora.

Na Creche 2, a coleta de dados ocorreu em uma sala de 60 m', contendo apenas um banco de madeira $(1,90 \times 0,30 \times 0,30 \mathrm{~m})$ e alguns colchonetes empilhados em um canto da sala, utilizados pelas crianças somente durante o sono pósalmoço.

\section{Equipamentos e Coleta de Dados}

Foram utilizadas duas câmeras fotográficas com funcionamento automático e simultâneo a cada 30 segundos, sem a presença do operador, as quais eram fixadas em suportes de madeira, preses no alto de paredes opostas. Cada duas fotos batidas simultaneamente, eras ampliadas em um mesmo papel, abrangendo todo o local. Foram obtidas de 30 a 40 fotos, por sessão.

Foram introduzidas, devido ao procedimento experimental, pequenas estantes de madeira $(1,10 \times 0,30 \times 0,50 \mathrm{~m}), 10$ na Creche 1 e 8 na Creche 2. Quatro estantes eram mal estruturadas, contendo uma papelão grosso na parte posterior, desde os pés até acima do tampo, sendo que duas dessas estantes em um grupo e uma no segundo grupo, tinham um outro papelão em uma das laterais, formando uma quina.

\section{Procedimento}

O estudo de CAMPOS-DE-CARVALHO (1989; CAMPOS-DE-CARVALHO \& ROSSETT: FERREIRA, 1993) constou de três fases, cada uma contendo uma estruturação diferente de espaço existente. A Figura I mostra uma ilustração do arranjo espacial da sala do Grupo 2 em cada uma das fases. Indica também a ampliação no mesmo papel das fotos obtidas simultaneamente pelas duas câmeras fotográficas, onde a parte central da sala aparece nas duas fotos.

\section{Fase I - Arranjo espacial aberto: habitual.}

Foram realizadas quatro sessões em um período de quatro semanas na Creche 1 e de duas semanas na Creche 2, onde o local era caracterizado por um espaço amplo e vazio.

\section{Fase II - Arranjo espacial aberto: introdução de pequenas estantes.}

Em ambas as creches foram realizadas seis sessões por um período de três semanas, após cerca de quinze dias de familiarização às estantes, introduzidas nas laterais do local para preservar 0 arranjo aberto; elas se fizeram necessárias para a montagem de zonas circunscritas na fase subseqüente.

Fase 111- Arranjo espacial semi-aberto: montagem de zonas circunscritas.

Nesta terceira fase a estruturação espacial caracterizou-se pela presença de duas zonas circunscritas (ZC1 e ZC2) montadas com as estantes da Fase 11. ZCI era mais estruturada (estantes com e sem papelão e em maior número; característica de fechamento mais saliente, com o quarto lado possuindo uma pequena abertura para a pas- 
sagem de crianças), localizando-se longe da área de permanência da educadora. ZC2, menos estruturada e menor (delimitada em três lados dois lados por duas estantes sem papelão e o terceiro, por uma parede), localizava-se perto da área da educadora. Foram realizadas seis sessões em um período de três semanas na Creche 1 e de cinco semanas na Creche 2, após cinco dias de familiarização ao novo arranjo.

\section{Aálise de Dados}

Para o Icvantamento dos dados do presente estudo utilizou-se uma máscara de acetato quadriculada, onde cada quadrado correspondia a $1 \mathrm{~m}^{2}$; quando colocada sobre as fotos, permitia registrar a formação de agrupamentos infantis. Estes foram diferenciados em agrupamentos entre crianças (somente com a presença de crianças) e entre criança(s) e adulto (agrupamento com a pre-

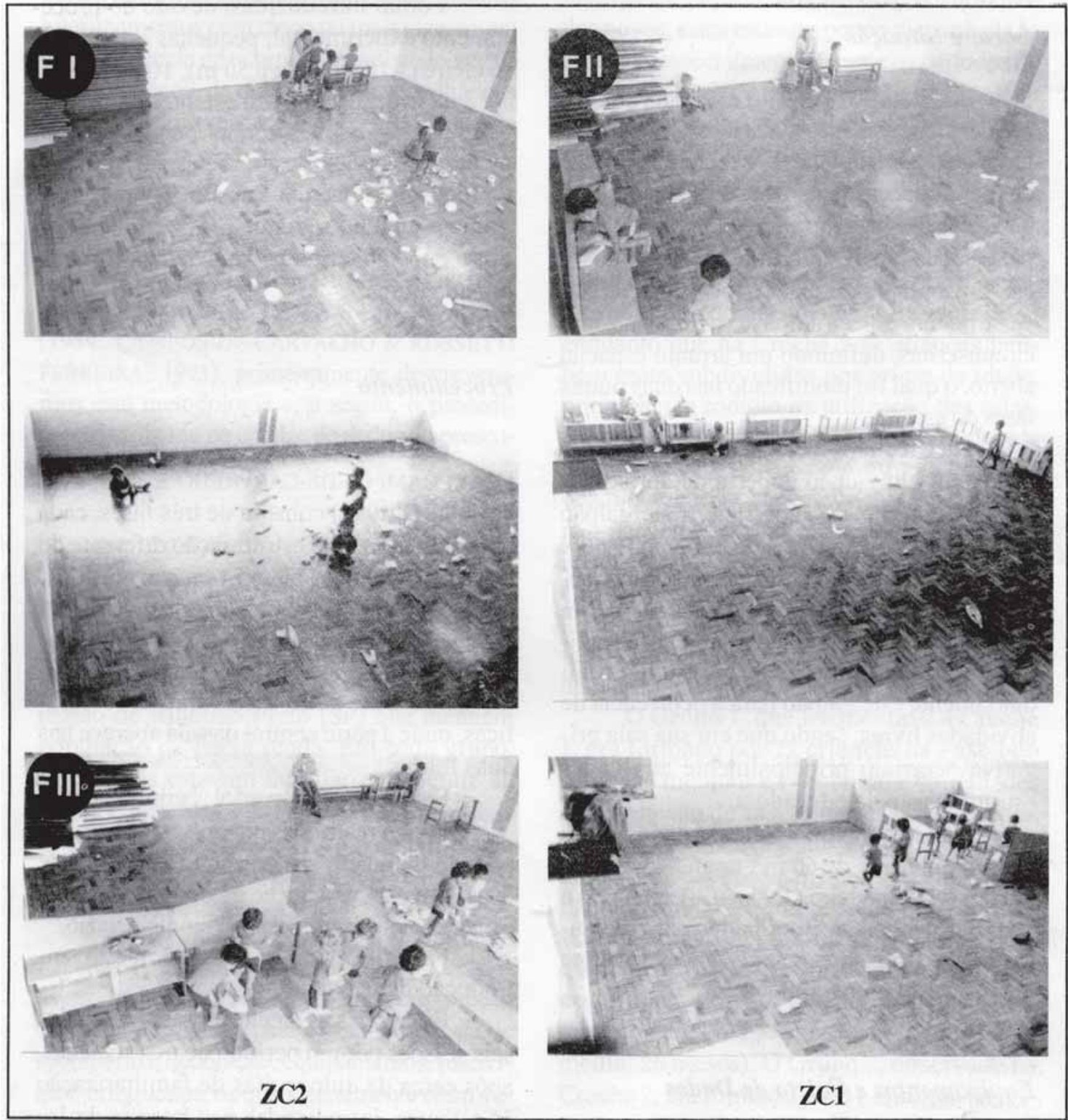

Figura 1. Fotos ilustrativas do arranjo espacial da sala do grupo 2 em cada uma das três fases (FI, FII, FIII). Em FIII, ZCI, indica a zona circunscrita maior e longe do adulto e a ZC2, a zona circunscrita menor e perto do adulto. 
sença da educadora, independentemente do número de crianças).

O critério utilizado foi o de proximidade física, onde dois, três, quatro, cinco ou mais elementos foram considerados agrupados quando estavam no máximo a $1 \mathrm{~m}$ de distância uns dos outros.

A cada foto, portanto a cada 30 segundos eram discriminados os tipos de agrupamentos observados - duos, trios, quadras ou agrupamentos com cinco ou mais elementos - e sua freqüência de ocorrência em cada sessão de cada fase. Este levantamento foi feito para cada uma das áreas identificadas em cada fase: Fase I - zona do adulto e o resto do local; Fase II - zona do adulto, estantes nas laterais do local e o resto do local; Fase III - zona do adulto, zonas circunscritas e o resto do local . Por zona do adulto entende-se a área de permanência habitual da educadora, sendo considerado cerca de $1 \mathrm{~m}$ ao seu redor; de acordo com a divisão da área em $\mathrm{m}^{2}$, a zona do adulto corresponde ao quadrado no qual o adulto se encontra e aos oito quadrados adjacentes a este.

\section{RESULTADOS}

Os dados sao apresentados para o conjunto das sessões de cada fase, porém as mesmas tendências foram observadas na maioria delas. Como as crianças componentes do Grupo I nas Fases II e III não eram as mesmas da inicial, com excecão de duas, a comparação dos resultados obtidos na Fase I com as subseqüentes é mais sugestivo que conclusivo.

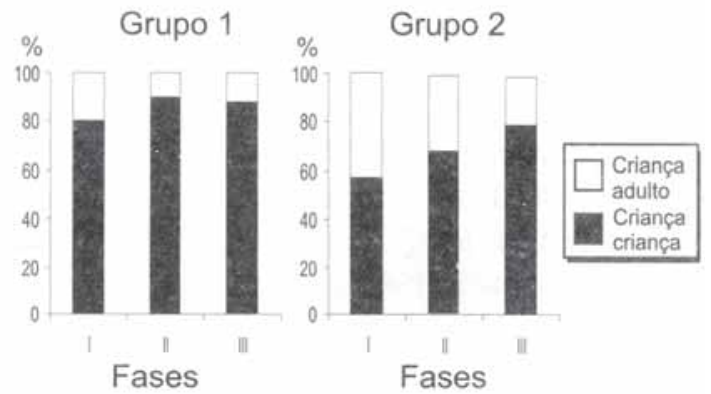

Figura 2 - Porcentagem de agrupamentos criança - adulto e criança - criança em cada fase.

A Figura 2 mostra a porcentagem total de agrupamentos ocorridos entre crianças e entre criança e adulto em cada fase do estudo, para ambos os grupos.

A Figura 2 evidencia que, para quaisquer das fases dos dois grupos, a ocorrência de agrupamentos entre as crianças é acentuadamente maior que os formados com o adulto. Ademais, observase que à medida que o espaço foi se tornando mais estruturado, da Fase I para a III, houve um aumento na ocorrência dos agrupamentos criança-criança, enquanto que os formados com a educadora decresceram, salientando-se o Grupo 2.

A Tabela I mostra a porcentagem de ocorrência de agrupamentos entre crianças nas três fases do estudo, especificando-os quanto ao número de componentes.

Observa-se pela Tabela I que, em ambos os grupos, as díades foram bem mais freqüentes, principalmente no Grupo I, sendo que à medida que aumentava o número de elementos nos agru-

Tabela 1. Porcentagem de ocorrência de agrupamentos criança-criança em cada fase

\begin{tabular}{|c|c|c|c|c|c|c|c|c|}
\hline \multirow[b]{3}{*}{ Agrupamentos } & \multicolumn{6}{|c|}{ Fases } & \multirow{2}{*}{\multicolumn{2}{|c|}{ Total }} \\
\hline & \multicolumn{2}{|c|}{ I } & \multicolumn{2}{|c|}{ II } & \multicolumn{2}{|c|}{ III } & & \\
\hline & G1 & G2 & G1 & G2 & G1 & G2 & G1 & G2 \\
\hline Duos & 78 & 79 & 76 & 69 & 68 & 67 & 74 & 70 \\
\hline Trios & 20 & 14 & 18 & 26 & 23 & 24 & 20 & 22 \\
\hline Quadras & 2 & 6 & 4 & 4 & 8 & 8 & 5 & 6 \\
\hline Cinco ou + & - & - & 1 & 2 & 2 & 1 & 1 & 1 \\
\hline Total & $23(230)^{*}$ & $19(156)^{*}$ & $39(396)^{*}$ & $36(298) *$ & $38(391)$ & $15(368)^{*}$ & & \\
\hline
\end{tabular}

* Frequência de agrupamentos.

* Tratamento estatístico dos dados - consulta a especialista em aplicação da estatística à área da psicologia indicou a utilização de estatística descritiva, em termos proporcionais (por exemplo, porcentagem) para permitir comparação entre os resultados obtidos. 
pamentos a porcentagem de ocorrência diminuta consideravelmente, especialmente naqueles com mais de quatro elementos. Ou seja, agrupamentos maiores ocorreram em menor freqüência que os menores. Para ambos os grupos, considerando as três fases conjuntamente, a porcentagem de diades é maior (cerca do triplo) que a porcentagem das tríades, as quais por sua vez, ocorreram cerca de quatro vezes mais que as quadras. Agrupamentos com cinco ou mais crianças raramente ocorreram e somente nas duas últimas fases.

Englobando todos os tipos de agrupamentos, a Tabela l evidencia maior ocorrência de agrupamentos nas duas últimas fases em relação à Fase I, salientando-se o Grupo 2, o qual mostrou um aumento considerável de uma fase para outra
- na Fase II, quase o dobro em relação à fase anterior e na Fase III, maior que o dobro dos agrupamentos observados na Fase I. Foi incluída nesta tabela? e na próxima? a freqüência total de agrupamentos (indicada entre parênteses), para mostrar como sua ocorrência foi elevada.

Com relação aos agrupamentos criançaadulto, a Tabela 2 mostra sua ocorrência nas três fases, especificando os tipos de agrupamento quanto ao tamanho.

Englobando todos os tipos de agrupamentos, verifica-se pela Tabela 2 que a porcentagem de agrupamentos criança-adulto é semelhante nas três fases, havendo um pequeno decréscimo na Fase II do Grupo 1 e na Fase III do Grupo 2, em comparação às outras fases.

Tabela 1. Porcentagem de ocorrência de agrupamentos criança-adulto em cada fase

\begin{tabular}{|c|c|c|c|c|c|c|c|c|}
\hline \multirow[b]{3}{*}{ Agrupamentos } & \multicolumn{6}{|c|}{ Fases } & & \\
\hline & \multicolumn{2}{|c|}{ I } & \multicolumn{2}{|c|}{ II } & \multicolumn{2}{|c|}{ III } & \multicolumn{2}{|c|}{ Total } \\
\hline & Gl & G2 & G1 & G2 & $\mathrm{Gl}$ & $\mathrm{G} 2$ & Gl & G2 \\
\hline Duos & 58 & 27 & 73 & 42 & 87 & 59 & 73 & 42 \\
\hline Trios & 26 & 26 & 20 & 42 & 9 & 26 & 18 & 33 \\
\hline Quadras & 7 & 28 & 7 & 12 & 4 & 7 & 6 & 16 \\
\hline Cinco ou + & 9 & 18 & - & 4 & - & 7 & 3 & 9 \\
\hline Total & $36(57)^{*}$ & $3(119)^{*}$ & $29(45)^{*}$ & $9(140)^{*}$ & $35(55)^{*}$ & $27(98)^{*}$ & & \\
\hline
\end{tabular}

* Freqüência de agrupamentos.

Nota-se que, para ambos os grupos, os agrupamentos diádicos foram bem mais frequentes que os demais, principalmente no Grupo I, havendo um decréscimo na porcentagem de ocorrência à medida que aumenta o número de participantes nos agrupamentos, tal como observado nos agrupamentos entre crianças. Salienta-se o Grupo 1, onde os duos ocorreram com uma frequência quatro vezes maior que os trios, considerando as três fases conjuntamente. Agrupamentos com cinco ou mais elementos (e quadras no Grupo 2) foram mais frequentes na Fase 1.

Além dos agrupamentos criança-adulto decrescerem da Fase I para III (mostrado na Figura 2), pudemos verificar que ocorreram predomi- nantemente na zona do adulto, em quaisquer das fases. Desta maneira, somente os agrupamentos entre crianças serão diferenciados quanto à ocorrência em áreas especificas a cada fase.

A Figura 3 permite verificar as relações entre as fases quanto à porcentagem de ocorrência dos agrupamentos diádicos e aqueles formados por três ou mais crianças, nas diversas áreas em cada fase. Como já apontado, a díades ocorreram em porcentagem bem maiores que os demais agrupamentos, os quais por sua vez ocorreram em porcentagem bastante baixa, especialmente os agrupamentos com mais de quatro elementos. Devido a esta distribuição, foram somadas as frequências de ocorrência dos trios, 
quadras e dos agrupamento com cinco ou mais crianças, denominado de agrupamentos com três ou mais crianças, facilitando assim a visualização comparativa com a díades.

Verifica-se pela Figura 3 que em ambos os grupos, excetuando-se a Fase I, os agrupamentos ocorreram com porcentagem maior nas áreas mais estruturadas, relação esta mais evidente para os agrupamentos con três ou mais crianças.
Na Fase 1, caracterizada por um espaço amplo e vazio, a zona do adulto era a única área estruturada do local, devido à presença da educadora. Os agrupamentos com três ou mais crianças foram mais freqüentes nesta área, salientando-se o Grupo 2 (GI = 56\%; G2 $=75 \%)$ Já as díades ocorreram em maior porcentagem no resto da sala (61\% no Grupo 1 e 54\% no Grupo 2).

Figura 3. Porcentagem de agrupamentos diádicos (duos) e com três ou mais criancas (3+), em áreas especificas a cada fase.

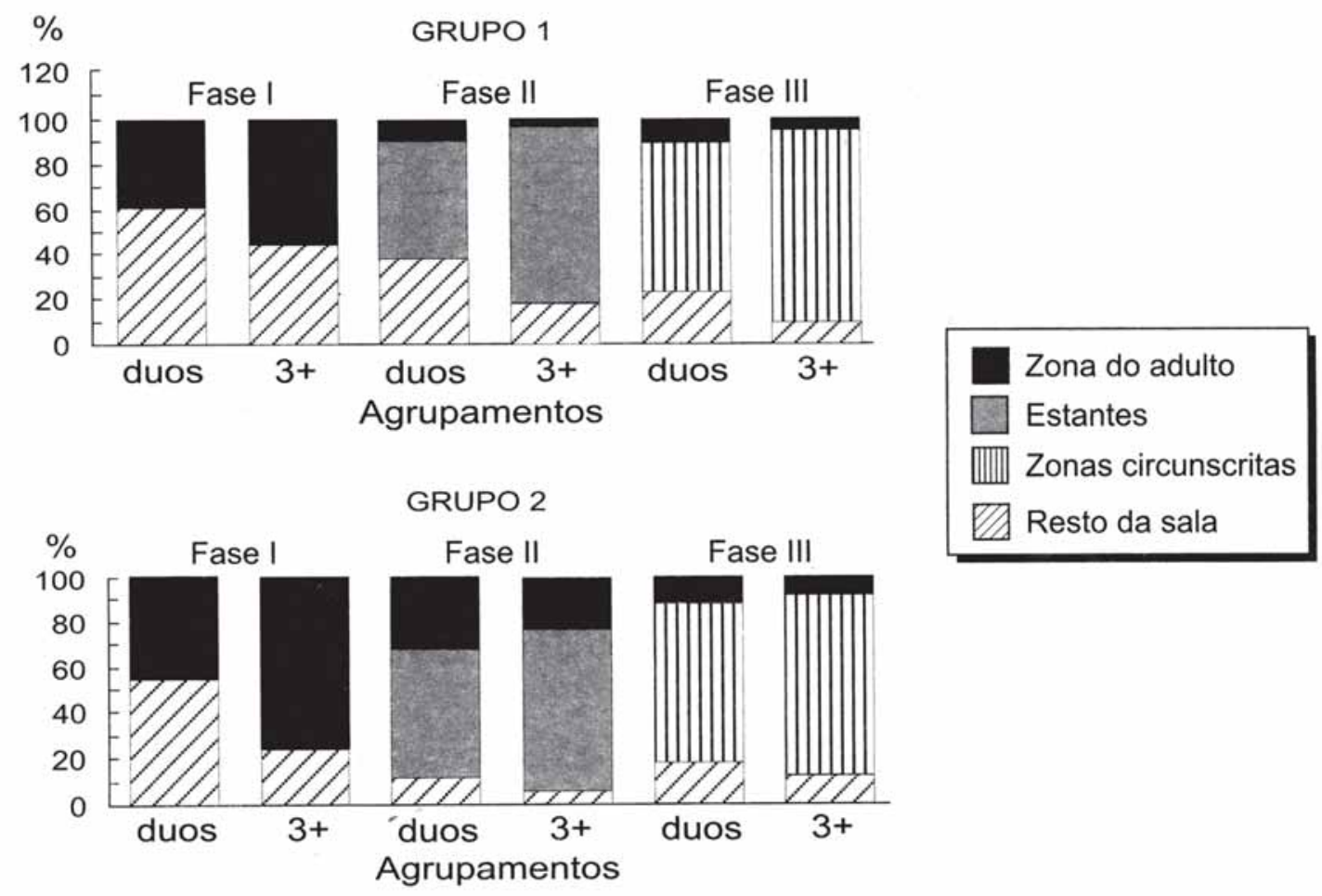

Na Fase 11, ainda caracterizada por um arranjo aberto, foram introduzidas as estantes de madeira. A Figura 2 evidencia que na área em volta das estantes há maior ocorrência de agrupamentos, tanto os diádicos (53\% no Grupo I e 57\% no Grupo 2) e, mais especialmente, os com três ou mais crianças (80\% no Grupo I e $72 \%$ no Grupo 2).

Na Fase III observa-se que as díades e os agrupamentos com 3 ou mais crianças, em ambos os grupos, ocorreram em maior porcentagem nas áreas mais estruturadas, ou seja, nas zonas circunscritas salientando-se os agrupamentos com 3 ou mais crianças (duos: G1 = 66\%; G 2 = 71\%. Agrupamentos com 3 ou mais: G $1=86 \%$; G 2 = 80\%).

\section{DISCUSSÃO}

A proximidade física tem sitio apontada como importante para a ocorrência de interações entre crianças, sobretudo entre as pequenas, sendo necessária para a ocorrência de formas mais simples de interações, por exemplo nas especulares, onde as crianças demonstram sua disposição para brincarem juntas através de ações simultaneas e seinelhantes (CAMAIONI, 1980; PEDROSA \& FAECÃO, 1993). Em seu estudo, PEDROSA \& FA CÃO ( 1993 ) apontam que a proximidade física foi necessária para estruturar brincadeiras de crianças no início do terceiro ano de vida, pois como a linguagem oral é ainda incipiente, as pistas visuais predominam na organização da brin- 
cadeira e o estar próximo ao outro facilita o contato. Já no final do terceiro ano, a proximidade física não é mais tão necessária, pois as crianças utilizam também pistas verbais na estruturação das brincadeiras, onde ações complementares se fazem presentes, determinadas principalmente pelo tema da brincadeira.

Embora seja um dos indicadores mais simples propostos para a análise de interacão de crianças, o critério proximidade física tem sido utilizado na análise de dados de grupos infantis (CARVALHO, 1992; HINDE \& ROPER, 1985), contribuíndo para uma compreensão dos mesmos (CARVALHO,1992).

CAMPOS-DE-CARVALHO \& RUBIANO (1996b), em um estudo metodológico, analisaram e compararam os dados de quatro sessões do Grupo 2 do presente estudo (durante a Fase III), coletados simultaneamente por câmeras fotográficas e de videoteipe. Proximidade física distância de até $1 \mathrm{~m}$ - foi o critério utilizado na análise das associações entre pares de crianças, via fotos, e atividade compartilhada engajamento na mesma atividade e orientação mútua na análise pelos vídeos. Embora as associações indicadas pela proximidade física em geral excederam aquelas por compartilhamento de atividades, semelhanças entre as análises apontam que as crianças geralmente envolveram-se em atividades compartilhadas quando próximas. Tais resultados fornecem uma base empírica para utilização da proximidade física como um bom indicador para uma análise global das associações ocorridas dentro de um grupo de crianças de 2 anos.

Em um outro estudo (CAMPOS DE CARVALHO e col., 1996), utilizando o critério de proximidade física para a análise de pares infantis dos mesmos dois grupos de crianças do presente estudo, demonstraram que, em ambos os grupos e em cada fase, houve uma porcentagem bem maior de crianças agrupadas do que isoladas; esta porcentagem aumentou gradualmente da Fase I para a III, à medida que o espaço foi se tornando mais estruturado fisicamente.

Nossos dados apontam que este acréscimo deveu-se à ocorrência crescente de agrupamentos entre crianças da Fase I para III, evidenciando o papel de suporte do arranjo espacial para a formação de agrupamentos entre crianças pequenas. Apesar dos evidentes progressos nas habilidades de crianças no terceiro ano de vida (CAMAIONI, 1980; HOMBERG, 1980) para estabelecer e manter interações com coetâneos, elas ainda necessitam muito de suportes ambientais. Nossos dados, corroborando os de LEGENDRE (1987, 1989; LEGENDRE \& FONTAINE, 1991), apontam o arranjo espacial, como um dos suportes ambien- tais para a ocorrência de contatos entre crianças no terceiro ano de vida.

Esta análise responde positivamente à primeira questão elaborada no início deste trabalho, onde nosso interesse era saber se, à medida que o espaço tornou-se mais estruturado (da Fase I para a III), haveria diferença no total de agrupamentos formados entre crianças e entre criança-adulto. Ademais, os agrupamentos formados com a educadora ocorreram, em quaisquer das fases, predominantemente na zona do adulto, enquanto que os agrupamentos entre crianças diferenciaram-se de fase para fase. É interessante salientar que as quadras e os agrupamentos com cinco ou mais elementos ocorreram com porcentagem um pouco mais elevada quando a educadora integrava o grupo, especialmente na Fase I, em comparação aos agrupamentos formados somente entre crianças; tal fato mostra o papel significativo do adulto para a formação de agrupamentos maiores entre crianças pequenas.

Os agrupamentos entre crianças ocorreram mais freqüentemente nas áreas mais estruturadas, específicas a cada fase - zona do adulto na Fase I (relação válida para os agrupamentos maiores), estantes na Fase II e zonas circunscritas na Fase III - respondendo assim positivamente à segunda questão elaborada no início do trabalho, ou seja, há diferença no total de agrupamentos formados em áreas específicas em cada fase. Chama atenção o papel de suporte exercido pelas áreas mais estruturadas para a ocorrência de agrupamentos com maior número de crianças, o que foi evidente em todas as fases na comparação entre díades e agrupamentos com três ou mais crianças.

Na Fase I a zona do adulto era a área mais estruturada do galpão (Creche 1) e da sala (na Creche 2) - o fato de agrupamentos com três ou mais crianças, em comparação aos diádicos, ocorrerem mais freqüentemente nesta zona, evidencia mais uma vez a importância do adulto para a formação de agrupamentos com maior número de crianças, especialmente em um tipo de arranjo espacial aberto, com escassez de materiais, equipamentos e mobiliários, tal como o que caracterizava o arranjo espacial da Fase 1. Já, a presença de estantes nas laterais do local (Fase II) e na formação de zonas circunscritas (Fase III), mostrou exercer um papel de suporte para a formação de agrupamentos entre crianças, especialmente os formados por três ou mais crianças, o que levou a uma diminuição considerável de agrupamentos na zona do adulto.

Na Fase II as estantes, únicos equipamentos acessíveis às crianças em um espaço amplo e vazio e com escassez de objetos, tornaram-se pólos atrativos para as crianças. Estas utilizavam a 
superfície de apoio das estantes para colocação e manipulação de objetos e como suporte para o próprio corpo, para sentar, deitar, ficar em pé, pular para o chão e novamente subir na estante. Tal análise está de acordo com uma visão sistêmica de desenvolvimento, evidenciando a interdependência entre o papel do adulto, a estruturação espacial, a escassez de objetos e equipamentos, em interação com as habilidades sociais de crianças pequenas.

Na Fase III, as zonas circunscritas montadas com as estantes exerceram um papel de suporte para a formação de agrupamentos entre as crianças, indo a favor dos dados obtidos por LEGENDRE ( 1987, 1989; LEGENDRE \& FONTAINE, 1991).

As zonas circunscritas facilitam a promoção e manutenção de interações entre coetâneos, fornecendo um sentido de proteção e privacidade; facilitam muito mais a continuidade de atividades que em arranjos abertos, por reduzirem a probabilidade de interrupções por outras crianças ou mesmo pelo adulto. Desta maneira, a zona circunscrita favorece à criança focalizar a atenção não somente em sua atividade, mas também na do companheiro, passo essencial para a ocorrência de atividades compartilhadas.

RUBIANO \& ROSSETTI-FERREIRA (1995), ao analisarem pelo vídeo as atividades desenvolvidas no arranjo semi-aberto pelos mesmos grupos de crianças do presente estudo, constataram que as zonas circunscritas favoreceram a ocorrência de brincadeiras, sobretudo as de "faz-deconta" (brincar de casinha, fazer comidinha, comer, beber, deitar o nenê, etc.). As autoras comentaram que as estantes criaram um cenário para as brincadeiras, por possibilitarem a reprodução de ações as quais, geralmente em nossa cultura, ocorrem em superficies elevadas e delimitadas.

Na cultura ocidental a criança, desde pequena, está em contato com mobiliários que possuem superficie de apoio (mesas, cadeiras, camas, sofás, estantes, etc.), sobre os quais rotineiramente se desenvolvem uma série de atividades comuns à cultura. Experiências já vivenciadas pelas crianças em superficies de apoio, tais como as estantes utilizadas na delimitação das zonas circunscritas, facilitariam o desenvolvimento conjunto de atividades. CAMPOS-DE-CARVALHO e RUBIANO (1996a, p. 122) apontam que as zonas circunscritas possibilitam “a re-apresentação no faz-deconta de atividades cotidianas (...) as quais funcionam como temas conhecidos pelos parceiros, em torno dos quais as crianças mantêm-se associadas, com possibilidade de constituírem-se na e pela interação com outras crianças tão jovens quanto elas”.
CARVALHO ( 1992) ilustra com episódios claros o papel desempenhado pelo compartilhamento de um conhecimento, na interação social de crianças pré-escolares. A posse de um conhecimento compartilhado oferece elementos para o desenvolvimento de brincadeiras conjuntas. como também para a construção de novos elementos compartilhados e até possibilitando, ou acentuando, a seletividade de parceiros.

LEGENDRE (1989; LEGENDRE \& FONTAINE, 1991) também apontam a importância deste aspecto, denominado por eles de rotinas pré-verbais, na interação de crianças menores de três anos. Comentam a importante função adaptativa destes sistemas transitórios de comunicação, para a integração de crianças pequenas em ambientes coletivos, durante o período no qual a comunicação verbal ainda não é predominante. Apontam que, além da imitação recíproca, o significado compartilhado de rotinas pré-verbais favorece o desenvolvimento de atividades compartilhadas, auxiliando no estabelecimento e manutenção de interações de crianças pequenas.

Em resumo, as zonas circunscritas delimitadas com as estantes favorecem o estabelecimento e manutenção de interações entre coetâneos, pois fornecem proteção e privacidade e facilitam o desenrolar de uma rotina de ações, as quais geralmente ocorrem sobre superfícies de apoio, propiciando maior oportunidade para o desenvolvimento mais continuo de atividades compartilhadas. Em um estudo subseqüente (MINGORANCE \& CAMPOS-DE-CARVALHO, no prelo), examinaram a questão da atração pela zona circunscrita, investigando especificamente o papel de suporte da variável circunscrição e da variável superficie de apoio, considerando que desde a Fase II as estantes exerceram um papel de suporte para a formação de agrupamentos entre as crianças. A análise da distribuição espacial das crianças evidenciou ocupação preferencial da zona circunscrita com apoio, em detrimento da zona circunscrita sem superficie de apoio e de estantes com superficie de apoio, porém sem circunscreverem uma zona.

O presente estudo evidenciou, nas três fases. maior ocorrência de agrupamentos em díades, indo a favor da literatura (STAMBAK \& VERBA, 1986). Embora durante o terceiro ano de vida as crianças já apresentam maior capacidade para iniciar e manter contatos com outra criança e/ou com o adulto, elas ainda encontram dificuldades para interagir simultaneamente com vários coetâneos, visto que neste período suas habilidades verbais e sociais estão se desenvolvendo. Destas considerações decorre a importância da organização do ambiente físico (do qual o arranjo espacial é uma das 
variaveis), para facilitar a ocorrência de interações entre crianças pequenas e com o adulto.

Ern suma, dois pontos se salientam no presente estudo: (1) o papel de suporte do arranjo espacial, mais especificamente das áreas mais estruturadas de cada fase, nas quais se observou a ocorrência preferencial de agrupamentos, principalmente daqueles formados por maior número de crianças; (2) o papel de suporte desempenhado, conscientemente ou não, pela educadora para a formação de agrupamentos entre crianças, o qual parece ser mais forte quanto menos estruturado está o espaço físico, desde que a ocorrência de agrupamentos entre crianças, na área em torno do adulto, diminuiu sensivelmente à medida que o espaço tornou-se mais estruturado.

O presente estudo contribui para apontar a relevância da variável arranjo espacial no contato entre crianças e entre crianças e adulto, na faixa etária de 2-3 anos em creches, colaborando assim para uma melhoria da qualidade do atendimento de crianças pequerias em creches e pré-escolas. De acordo com nossa perspectiva cabe ao adulto a responsabilidade pela estruturação e organização contínua do ambiente, para favorecer o envolvimento das crianças em atividades compartilhadas com os companheiros sem a intervenção direta do adulto, propiciando às crianças, conseqüentemente, maior oportunidade de interação com a própria educadora. Esta torna-se mais disponível para observar as ações das crianças e estabelecer um contato mais individualizado com aquelas que perceba necessitarem de uma atenção especial, ou desenvolver atividades com um pequeno subgrupo de crianças, possibilitando-lhe assim atuar diretamente junto à criança como um interlocutor privilegiado. Por exemplo, a estruturação do espaço em um arranjo semi-aberto permite ao educador assuinir este papel, enquanto que em um arranjo aberto as crianças permanecem em sua volta, solicitando constantemente sua atenção, sendo pouca a disponibilidade do adulto para atendê-las, visto o número grande de crianças sob sua responsabilidade.

Desta maneira, o arranjo espacial, assim como outras variáveis do ambiente fisico e social, deve também ser considerado no planejamento de ambientes infantis, especialmente os coletivos. Entretanto, via de regra as caracterís- ticas físicas de um ambiente, infelizmente, têm sido negligenciadas, exceto pelas recomendações gerais de que esses ambientes devem ser ricos, estimuladores e seguros, evidenciando um ponto de vista de que os componentes físicos de um ambiente são considerados como um cenário sem grande importância. Ademais, concepções dos adultos sobre o desenvolvimento infantil guiam, conscientemente ou não, a organização e ambientes em instituições educacionais. Por exemplo, conceber o desenvolvimento da criança centralizado no adulto e, conseqüentemente, perceber a criança, sobretudo a pequena, como necessitando de direção e orientação quase constante do adulto, facilitaria ao adulto, para desempenhar suas funções em um ambiente coletivo, estruturar o local com um amplo espaço central e vazio - arranjo espacial aberto - típico de nossas creches, especialmente durante os períodos de atividades livres. Assim sendo, a maneira de ver o desenvolvimento da criança, pode favorecer ou dificultar, por exemplo através da estruturação espacial, a ocorrência de interações não só entre as crianças como também com o próprio educador (CAMPOS-DE-CARVALHO \& RUBIANO, 1996a).

Destas considerações depreende-se a importância e necessidade de se trabalhar estes aspectos, dentre muitos outros, com os educadores infantis, em programas de formação, tanto prévia como em serviço. Como apontado por VITORIA (1997), a formação do educador infantil e a maneira mais eficaz para garantir a melhoria da qualidade do atendimento oferecido, visando condições melhores para um adequado desenvolvimento das crianças, desde que o adulto é o principal mediador do desenvolvimento infantil; além de conteúdos técnicos, os programas de formação deveriam incluir tópicos sobre a história de vida, crenças e concepções do educador desenvolvidos em suas relações sociais e em sua experiência profissional.

\section{AGRADECIMENTOS}

À FAPESP (Proc. 91/3584-2; 96/2064-9) e CNPq (Proc. 300750/93-5; 522913/95-4) pelos auxílios recebidos. 


\begin{abstract}
This study analyzed the formation of peergroups and caregiver-children groups (groups of two, three, four and five or more components), using the same data collection of a previous study carried out with groups of 2-to-3-year-old children from two day care centers which serve the low income families in the Ribeirão Preto (SP) area. The data were collected by two automatic photographic cameras shooting at every 30 seconds. The procedure included three phases: I open anrangement: the usual broad and empty space (4 sessions); II - open arrangement: inclusion of small shelves along the periphery of tho space (6 sessions); III - semi-open arrangement: formation of two circumscribed zones, areas delimited on at least three sides by small barriers (6 sessions). Using a criterion of physical proximity of $1 \mathrm{~m}$, the analysis showed: (1) an increase in the occunrence of peer groups and a decrease in adult-children groups with increased spatial structure, (2) dyadic children groups were more frequent, and (3) a preferential occunrence of peer groups, especially with three or more children, in the more structured areas of each phase, adult's zone (Phase I), shelves (Phase II) and circumscribed zones (Phase III). These data indicate the relevance of spatial arrangements for the formation of peer groups and for designing children's collective environments.
\end{abstract}

Key-words: peer groups, spatial arrangement, day care centers, physical proximity.

\section{REFERENCIAS BIBLIOGRÁFICAS}

BRONFENBRENNER, U. Toward an experimental ecology of human development. Am. Psychol. 513-531, 1977.

BRONFENBRENNER, U.; CROUTER, A. C. The evolution of environment modes in development research. In: MUSSEN, P. H. Ed., Hand book of child psychology. New York: Wiley, V. l, p. 358-414, 1983.

CAMAION I, L. L. Interacione tra bambini. Roma, Armando Ammando, 19X0.

CAMPOS-DE-CARVALHO, M. I. Organização espacial da área de atividades livres em creches. In: $18^{a}$ Reunião Anual de Psicologia da Sociedade de Psicologia de Ribeirão Preto, Ribeirão Preto (SP), 1988. Anais Ribeirão (SP), 1989, p. 305-310.

CAMPOS-DE-CARVALHO, M. I. Psicologia ambiental - algumas considerações. Psicol. Teor Pesq, 2: 435-447, 1993.

CAMPOS-DE-CARVALHO, M. I., MENEGHINI, R.; MINGORANCE, R. C. Arranjo espacial e formação de pares entre crianças de 2-3 anos em creches. Psicol, 27(2): p. 117-137, 1996.

CAMPOS-DE-CARVALHO, M. I. ; ROSSETTI FERREIRA, M. C. Importance of spatial arrangements for young children in day care centers. Child Environ. 10: 19-30, 1993.

CAMPOS-DE-CARVALHO, M. I.; RUBIANO, M. R. B. Organização do espaço em instituições pré-escolares. In: OLIVEIRA, Z. M. R. Org., Educação infantil, mudos olhares. São Paulo, Editora Cortez, $3^{\text {a }}$ ed., 1996a. p. 107-130.
CAMPOS-DE-CARVALHO, M. I.; RUBIANO, M. R. B. Rede social de crianças pequenas em creche: análise por proximidade fisica e atividade companilhada. Psicol. Teor. Pesq. 12(2): 129-136, 1996b.

CARVALHO, A. M. A. Seletividade e vínculo na intenação entre crianças. São Paulo, 1992. [Tese de livre-docência - instituto de Psicologia da USP].

ECKERMAN, C. O.; STEIN, M. R. The toddler's emerging interactive skills. In: RUBIN, K. H.; ROSS, H. S. Eds. Peer relationship and social skills in childhoad. New York, SpringerVerlag, 1982. p. 41-71.

HINDE, R. A.; ROPER, R. Componement social dans un groupe de jeu: I'incidence des relations d’égal àégal. Bul. Psychl. 16 (383): 280-290, 1985.

HOMBERG, M. C. The development of social interchange patterns from 12 to 42 months. Child Dev. 51: 448-456, 1980.

LEGENDRE, A. Appropriation por les enfants de l'environment architectural. Enfance 3: 389395, 1983.

LEGENDRE, A. Transformation de l'espace d'activites et échanges sociaux de jeunes enfants en crèche. Psychol. Francaise, 32: 3143, 1987.

LEGENDRE, A. Young children's social competences and their use of space in day-care centers. In: SCHNEIDER, B. H., ATTILI, G., NADEL, J.; WEISSBERG, R. Eds., Social competence in developmental perspective. Holland, Kluwer, 1989, p. 263-276. 
LEGENDRE, A.; FONTAINE, A.M. The effects of visual boundaries in two-year-olds playrooms. Child. Environ. Q. 8: 2-16, 1991.

MINGORANCE, R. C.; CAMPOS-DE-CARVALHO, M. I. Distribuição espacial de crianças de 2-3 anos pela arca de atividades livres em creche. Rev. Interam. Psicol. [No prelo].

MOORE, G. T. Effects of the definition of behavior settings on children s behavior: A quasi-experimental field study. J. Environ. Psychol. 6: 205-231, 1983.

MOORE, G. T. The physical environment and cognitive development in day-care centers. In: WEINSTEIN, C. S.; DAVID, T. G. Eds., Spaces lor children: the built environment and child development. New York, Plenum, 1987, p. $41-72$.

MUELLER, E.; LUCAS, T. A developmental analysis of peer interaction among toddlers. In: LEWIS, M.; ROSENBLUM, L. A. Eds., Friendship and peer relations. New York, Wiley, 1975, p. 223-257.

PEDROSA, M. I.; FALCÃO, M. T. Play structures as cues to the transformation of the social space in young children. In: XII Biennial Meetings of Title International Society for the Study of Behavioural Development. Recife, Brazil, July, 1993.

ROSSETTI-FERREIRA, M. C. O apego e as reações da criança à separação da mãe. Cad. Pesq. 48: 3-19, 19 X4.
RUBIANO, M. R. B.; ROSSETTI FERREIRA, M. C. Interacción y juegos en las guarderias, el papel de la organización espacial. Rev. Latinoam. P.sycol. 27(3): 429-450, 1995.

STAMBAK, M.; VERBA, M. Organization of social play among toddlers: An ecological approach. In: MUELLER, E.C.; COOPER, C.R. (Eds.), Process and outcome in peer relationship. Orlando, Acadermic l'ress, I’)Xóó p. 2 3-295.

STOKOLS, D. Environmental psychology. Annual Rev. Psychol. 29: 253-295, 197X.

VALSINER, J. Culture and the development of childrens action - a cultural-historical theory of developmental psychology. New York, Wiley, 1987.

VITÓRIA, T. Representações de educadores sobre as mães e famílias das crianças da creche. Ribeirão Preto, 1997. [Dissertação de Mestrado - Programa de Pós-Graduação em Saúde Mcntal, Faculdade de Medicina de Ribeirão Preto da USP].

WOHLWILL, J. F. The confluence of environmental and developmental psychology: Signpost to an ecology of development? Hum. Dev. 23: 354-358, 1080.

WOHLWILL, J. F.; HEFT, H. The physical environment and the development of the child. In: STOKOLS, D.; ALTMAN, D. Eds, Handbook of environlmental psychology. New York, Wiley, 1987, p. 2XI-378.

Recebido em 29/03/98 Aprovado em 30/04/98 